\title{
Special issue: Computational intelligence models for image processing and information reasoning
}

\author{
Chee Peng Lim ${ }^{\mathrm{a}, *}$, Canicious Abeynayake ${ }^{\mathrm{b}}$, Mika Sato-Ilic ${ }^{\mathrm{c}}$ and Lakhmi C. Jain ${ }^{\mathrm{d}, *}$ \\ ${ }^{a}$ Deakin University, Burwood, VIC, Australia \\ ${ }^{\mathrm{b}}$ Defence Science and Technology Organisation, SA, Australia \\ ${ }^{\mathrm{c}}$ University of Tsukuba, Tsukuba, Japan \\ ${ }^{\mathrm{d}}$ University of South Australia, Mawson Lakes, SA, Australia
}

\begin{abstract}
Computational Intelligence (CI) models comprise robust computing methodologies with a high level of machine learning quotient. CI models, in general, are useful for designing computerized intelligent systems/machines that possess useful characteristics mimicking human behaviors and capabilities in solving complex tasks, e.g., learning, adaptation, and evolution. Examples of some popular CI models include fuzzy systems, artificial neural networks, evolutionary algorithms, multi-agent systems, decision trees, rough set theory, knowledge-based systems, and hybrid of these models. This special issue highlights how different computational intelligence models, coupled with other complementary techniques, can be used to handle problems encountered in image processing and information reasoning.
\end{abstract}

Keywords: Computational Intelligence, image processing, information reasoning, fuzzy models, membrane computing, Bayesian Belief Network

\section{Introduction}

In this special issue, a total of seven articles that cover various $\mathrm{CI}$ models for undertaking complex problems in two application domains, i.e., image processing (the first four articles) and information reasoning (the last three articles), are included. The main objective of this special issue is to highlight a small fraction of CI

\footnotetext{
${ }^{*}$ Corresponding authors. Dr. Lakhmi C. Jain, University of South Australia, Mawson Lakes, SA, Australia. E-mail: Lakhmi. jain@unisa.edu.au (Lakhmi C. Jain) and Dr. Chee Peng Lim, Deakin University, Burwood, VIC, Australia. E-mail: chee.lim@ deakin.edu.au (Chee Peng Lim).
}

models that are useful for image processing and information reasoning tasks, and it must not be treated as a comprehensive coverage of what CI models can do in these two application domains. A summary for each article is as follows.

A hierarchical model for template matching with a fuzzy metric and a decision tree is described in the first article [1]. Image features are mapped to a fuzzy plane and fuzzy conditions for approximate matching are utilized. A decision tree is then deployed to evaluate the feasibility of a block of the input image for matching with the template. Facial expression images are used to assess the applicability of the proposed model. The 
results show that the model is able to perform with a high matching accuracy rate with a low computational overhead.

A fuzzy model for fall detection and inactivity monitoring in infrared video is proposed in the second article [7]. A human detection algorithm is used to produce the necessary data for the fuzzy model to detect whether a genuine fall has occurred. An experimental study with a number of static and dynamic falls is conducted to demonstrate the usefulness of the proposed model in detecting falls, as well as to distinguish true and false falls.

In the third article [6], a new model based on a tissue P system and fuzzy entropy for multi-level thresholding in image segmentation is presented. The $\mathrm{P}$ tissue system with a special membrane structure is used to accelerate the convergence rate and to enhance diversity of object in the system, while fuzzy entropy is used as the evaluation criterion. The proposed model has been shown to be more effective than other evolutionary-based methods in terms of applicability and computational efficiency.

A multi-objective image segmentation model with an interactive evolutionary computation framework is described in the fourth article [5]. The multi-objective genetic algorithm is used to simultaneously optimize two parameters, i.e., the overall deviation and the connectivity measure, in image segmentation. The interactive evolutionary computation framework is then used to embed users' subjective evaluation in the optimization process. The experimental results show that the proposed model is able to produce segmented images with good quality as compared with those from other methods.

The fifth article [2] presents a fuzzy reasoning model that is useful for analyzing tourism information. In particular, seat availability data of bullet trains in Japan are used for analysis, and useful rules are derived from the data set. The fuzzy reasoning model can be used to effectively deal with ambiguous data as well as subjective information from experts. Performance comparison with a linear regression model shows the usefulness of the proposed method in analyzing tourism data.

A fuzzy inference system-based model for criterionreferenced assessment is introduced in the sixth article
[3]. A fuzzy rule selection scheme based on the genetic algorithm is used, and unselected rules are deduced using the approximate analogical reasoning schema in such a way that the monotonicity properties of the fuzzy inference system are preserved. A case study pertaining to assessment of laboratory projects by university students is used to show the applicability of the proposed model.

A framework for technical risk assessment of system integration is described in the seventh and last article [4]. The framework comprises a Bayesian Belief Network and other complimentary parametric models for risk assessment. Rationale and modeling objectives are presented and illustrated by examples. In addition, knowledge elicitation issues are also discussed.

The guest editors are grateful to the authors for their contributions, to the reviewers for their time and effort in reviewing the manuscripts, and to the journal production team for their support and help in producing this special issue.

\section{References}

[1] A. Halder, R. Mandal and A. Konar, A hierarchical algorithm for fuzzy template matching in emotional facial images, Journal of Intelligent \& Fuzzy Systems 24 (2013), 201-214.

[2] T. Hasuike and T. Ichimura, Web intelligence for tourism using railway data by a simplified fuzzy reasoning method, Journal of Intelligent \& Fuzzy Systems 24 (2013), 251-259.

[3] T.L. Jee, K.M. Tay and C.K. Ng, A new fuzzy criterionreferenced assessment with a fuzzy rule selection technique and a monotonicity-preserving similarity reasoning scheme, Journal of Intelligent \& Fuzzy Systems 24 (2013), 261-279.

[4] I. Loutchkina, L.C. Jain, T. Nguyen and S. Nesterov, The systems integration technical risk assessment fusing of Bayesian Belief Networks and parametric models, Journal of Intelligent \& Fuzzy Systems 24 (2013), 281-296.

[5] W.S. Ooi and C.P. Lim, Multi-objective image segmentation with an interactive evolutionary computation framework, Journal of Intelligent \& Fuzzy Systems 24 (2013), 239-249.

[6] H. Peng, J. Wang, M.J. Pérez-Jiménez and P. Shi, A novel image thresholding method based on membrane computing and fuzzy entropy, Journal of Intelligent \& Fuzzy Systems 24 (2013), 229-237.

[7] M.V. Sokolova, J. Serrano-Cuerda, J.C. Castillo and A. Fernandez-Caballero, A fuzzy model for human fall detection in infrared video, Journal of Intelligent \& Fuzzy Systems 24 (2013), 215-228. 